\title{
Il concetto di mimesi e la sua interpretazione nella letteratura
}

The concept of mimesis and its interpretation in literature

\author{
Karolina JANOWSKA ${ }^{1}$ \\ Ateneum-Szkoła Wyższa w Gdańsku \\ Mariusz HYBIAK ${ }^{2}$ \\ Uniwersytet Gdański/Miejska Biblioteka Publiczna im. Floriana Ceynowy \\ w Rumi
}

\begin{abstract}
Astratto
La mimesi come termine teorico e letterario si è formata già nell'antichità. Tentativi di definirlo sono già stati fatti da Platone nei suoi dialoghi, mentre fu solo Aristotele nella sua congeniale Poetica a formulare questo argomento in modo tale da diventare la base di discussioni e indagini scientifiche, molte delle quali continuano ancora oggi. La creatività mimetica ha dominato l'arte per centinaia di anni, raggiungendo l'apice della popolarità a cavallo tra il XIX e il XX secolo (sebbene siano emerse e funzionassero tendenze precedenti che quasi programmaticamente deviassero da questo percorso creativo; un esempio può essere l'arte dell'era romantica con tutti suoi aspetti mistici). Nel XX secolo, l'arte ha iniziato a cambiare in modo molto dinamico, seguendo lo sviluppo industriale e tecnologico incredibilmente rapido in Europa e nel mondo. Questo, a sua volta, ha portato allo sviluppo dinamico di nuove tendenze, nuovi modi di pensare l'arte.

1 https://orcid.org/0000-0002-5539-2318

Ateneum-University in Gdansk, Faculty of Modern Languages

k.janowska@ateneum.edu.pl

2 (P) https://orcid.org/0000-0002-5760-6906

Uniwersytet Gdański/ Miejska Biblioteka Publiczna im. Floriana Ceynowy w Rumi, Stacja Kultura

dkkrumia@gmail.com
\end{abstract}


Parole chiavi: mimesi, Aristotele, letteratura, arte, filosofia, poesia, Platone, concetto platonico

\begin{abstract}
Mimesis as a theoretical and literary term was already formed in antiquity. Attempts to define it have already been made by Plato in his dialogues, while it was only Aristotle in his congenial Poetics who formulated this topic in a way that became the basis for scientific discussions and inquiries, many of which continue to this day. Mimetic creativity dominated art for hundreds of years, reaching the peak of popularity at the turn of the 19th and 20th centuries (although earlier trends emerged and functioned that almost programmatically turned away from this creative path; an example could be art from the Romantic era with all its mystical aspects). In the 20th century, art began to change very dynamically, in a way following the incredibly fast industrial and technological development in Europe and around the world. This, in turn, resulted in the dynamic development of new trends, new ways of thinking about art.
\end{abstract}

Keywords: mimesis, Aristotle, literature, art, philosophy, poetry, Plato, Platonic concept

\title{
1. Il significato originale di mimesi
}

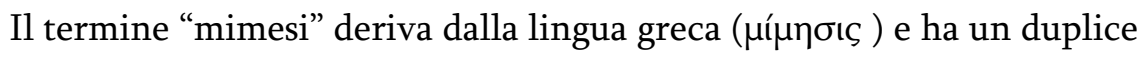
significato: può essere interpretato come un'imitazione (imitazione) o una somiglianza. Se volessimo fare riferimento strettamente alla definizione del dizionario, dovremmo supporre che la mimesi sia una "categoria estetica che significa imitazione della realtà da parte dell'arte". Questo è anche il caso, ma il concetto presentato in questo modo è molto limitato. Secondo un'altra definizione leggermente più ampia di mimesi significa:

(...) il termine (anche nella forma traslitterata mìmesis) viene usato soprattutto nel linguaggio filosofico, dove acquista importanza con Platone il quale con esso designa la somiglianza delle cose sensibili alle idee; nella concezione platonica dell'arte, la mimesi è da condannare perché, imitando le cose, che a loro volta sono copia delle idee, si allontana tre volte dal vero. Nell'estetica aristotelica, mimesi acquista un significato positivo, come imitazione della forma ideale della realtà, per cui l'operare dell'artista diventa simile all'operare della natura. Ripreso nella critica letteraria contemporanea, il termine indica generalmente la rappresentazione di una realtà ambientale, sociale, culturale, ecc., attuata perseguendo a varî livelli (ideologico, stilistico, documentario, ecc.) l'obiettivo di una riproduzione il più possibile realistica e impersonale di tali realtà (http://www.treccani.it/vocabolario/mimesi/).

Vale la pena citare altre definizioni che recitano come segue: 
1. imitazione della realtà e della natura che, secondo la concezione estetica classica, sarebbe a fondamento della creazione artistica | (non com.) imitazione,

2. nella filosofia platonica, il rapporto di imitazione che lega le cose del mondo sensibile, finite e particolari, alle realtà universali e sovrasensibili delle idee (https://www.garzantilinguistica.it/ricerca/ ?q=mimesi).

Per capire cosa fosse realmente mimesi, è necessario ritornare al concetto di mimesi nei significati ad essa attribuiti nell'antichità. Nel senso più primitivo, la mimesi era strettamente correlata all'arte espressiva (non imitativa), più precisamente - alla danza. Quindi la mimesi consisteva nell'esprimere emozioni, sperimentare in una parola-musica-danza (corea trina). Un attore avrebbe dovuto esprimere questi sentimenti. Un altro, più tardo, significato di mimesi si riferiva già alle arti che imitano la natura, e più specificamente alle leggi che la governano, e questo senso è introdotto da Democrito ed Eraclito (Tatarkiewicz, 1960, pp. 27-28). In questo approccio, la mimesi è un'imitazione (della natura). Dall'osservazione dei suoi diritti, l'uomo trae conclusioni che gli consentono di applicare queste leggi nella pratica (ad esempio la costruzione di nidi da parte degli uccelli può essere un modello e un'ispirazione per gli atti architettonici dell'uomo). L'essenza di questo concetto di mimetismo sembra essere la razionalità, o meglio l'assunzione di razionalità delle azioni umane, basata su schemi definiti da regole.

Quando si parla del concetto pitagorico, va sottolineato che va ben oltre le questioni puramente estetiche. Così, secondo l'opinione dei pitagorici, il mondo sensualmente percepibile imita il più alto nell'aspetto ontologico, il perfetto ordine numerico. La scoperta pitagorica nel campo della musica sembra essere l'esempio più importante di questo tipo di imitazione. Pitagora, usando uno strumento a corda singola chiamato monocordo, notò una stretta relazione matematica tra l'altezza e la frequenza delle vibrazioni e la lunghezza della corda. È così che ha scoperto gli intervalli. Quindi, se percepiamo un ordine nel mondo visibile, è perché riflette le più alte leggi matematiche. Questo concetto di mimesi presuppone la possibilità della partecipazione umana all'armonia dell'Universo: sperimentando e percependo la musica, possiamo partecipare a ciò che è cosmico (Krokiewicz, 2000, p. 97).

Nel senso più recente, la mimesi comincia a essere intesa come un'imitazione (copia, rispecchiamento) delle apparenze - in questa prospettiva 
si dovrebbero vedere Socrate e Platone. Le conclusioni di tale mimetismo sono perfettamente formulate da Reale (1996, p. 208):

1. l'arte non rivela la verità, ma la copre perché non la conosce;

2. non migliora un uomo, ma lo vizia perché mente;

3. non educa, ma rovina gli effetti dell'educazione, perché si rivolge alle facoltà irrazionali dell'anima, che sono le parti inferiori dell'uomo.

1. sztuka nie odkrywa prawdy, ale ją zakrywa, ponieważ nie poznaje;

2. nie czyni człowieka lepszym, ale go psuje, ponieważ jest kłamliwa;

3. nie wychowuje, lecz psuje efekty wychowania, ponieważ zwraca się do nierozumnych władz duszy, które są niższymi częściami człowieka.

Il concetto di mimesi era direttamente correlato alla concezione greca dell'arte, quindi, prima di procedere alla parte successiva dell'opera, in cui verrà presentato il concetto platonico e aristotelico di mimesi, è necessario richiamare il significato originario dei concetti legati all'arte e alla poesia utilizzati nell'antica Grecia. Oggigiorno, quando parliamo di arte, intendiamo principalmente belle arti (pittura, letteratura, musica, scultura). Questa comprensione è stata così saldamente stabilita in noi che il termine "bello" diventa inutile, perché è tautologico (se l'arte è - bella). Tuttavia, non bisogna dimenticare che per gli antichi (prima di Aristotele) la poesia non era affatto un'arte.

L'arte (techne) era intesa come un'attività umana basata su abilità e conoscenze fondate sulla conoscenza delle regole. Techne si riferiva quindi strettamente alla poiesis, cioè a tutta la produzione umana. L'arte nel senso primario è quindi ad es. l'arte della guarigione, tutti i mestieri (es. falegnameria, edilizia), e certamente anche - come diremmo oggi - arte culinaria. Per ovvie ragioni, però, non è un'arte il produrre che non si basa su conoscenze e regole, ma nasce da ciò che potremmo definire esperienza, abitudine, abilità, istinto, ispirazione.

Come dice Władysław Tatarkiewicz (1960, p. 39):

Secondo loro, l'arte era ciò che si poteva imparare e la poesia era ciò che non si poteva. La poesia, per intervento divino, dà la conoscenza del genere più elevato; guida le anime; educa le persone; le fa, o almeno può renderle migliori. L'arte fa qualcosa di completamente diverso: produce oggetti necessari, a volte perfetti. 
Sztuką było według nich to, czego można się nauczyć, a poezją - czego nie można. Poezja dzięki boskiej interwencji daje wiedzę najwyższego rodzaju; kieruje duszami; wychowuje ludzi; czyni, a przynajmniej może czynić ich lepszymi. Sztuka robi coś zupełnie innego: produkuje przedmioty potrzebne, niekiedy doskonałe.

In questo senso, la poesia diventa un depositario di tempi e tradizioni passati. Per quanto riguarda la verità trasmessa attraverso la poesia, le opinioni dei greci erano divise. Omero ed Esiodo cantarono il dono della poesia, credendo nella verità o almeno nella possibilità di verità dei suoi messaggi, mentre Solone e Pindaro vi vedevano una fabbricazione, persino un deliberato fallimento della verità.

Mimesi raccoglieva idee diverse. In letteratura, la mimesi è diventata sinonimo di finzione, creazione e costrutto artistico. Nell'antichità europea, la mimesi è principalmente l'imitazione di autori e testi. Nel Medioevo, St. Agostino ha affermato che l'imitazione riguarda qualcosa che è invisibile e non verificabile. Ha combinato Imitatio Christi con imitatio naturae (Mitosek, 1997, p. 153). Gli autori che si occupano del problema della mimesi parlano di mimesi linguistica (Ohmann, Bachtin), di mimesi formale (Słowiński), che la mimesi è una rappresentazione, una rappresentazione del mondo oggettivo nella letteratura (Auerbach, Lukács, Girard), che è un'imitazione di un testo (Mayenov). Per Blumenberg, la mimesi si esprime nella tendenza a costruire finzione (Kudra, 2007, p. 298).

Derrida tratta originariamente il problema della mimesi. Crede che l'arte sia creazione, imitazione di ciò che non c'è, cioè inventare. Derrida crede che non ci sia pura invenzione. Nel XX secolo la ricerca di somiglianze e corrispondenze tra l'arte e il mondo esterno era considerata un degrado dell'arte, l'imitazione si contrapponeva alla creazione.

Si può dire che la mimesi è uno strumento cognitivo, ontologico e sociologico di elaborazione delle informazioni, senza il quale l'intellettualizzazione non sarebbe possibile. Nello studio della letteratura, la mimesi è usata per descrivere il rapporto tra arte e natura (realtà - anche se diversamente intesa). Nel caso dell'imitazione in un'opera letteraria, abbiamo a che fare con un trasferimento, cioè il trasferimento di idee da una sfera all'altra - qui dal reale al fittizio. Nella letteratura realistica, il problema del mimetismo è ridotto a un alto grado di probabilità, veridicità, rappresentatività e tipicità. 


\section{Mimesi nella conclusione di Platone e Aristotele}

Fin dalle sue origini, nelle opere e negli scritti di filosofi come Aristotele o Platone, l'idea di mimesi è stata prominente nell'estetica occidentale. La teoria dei classici greci sull'arte considerava già lo sforzo artistico come un'imitazione della realtà. Queste imitazioni, dal punto di vista aristotelico e platonico, non dipendono dalla cognizione per esistere, a differenza degli elementi di finzione che dipendono da detta cognizione affinché gli oggetti esistano. La metafora dell'arte come specchio della natura materializza la mimesi. Questo concetto, emerso nei primi dialoghi di Platone, è secondo Abrams (1974), probabilmente la teoria estetica più primitiva, dove l'arte, come le ombre e le immagini nell'acqua o nello specchio, prende le distanze dal mondo delle idee. Tuttavia, a differenza della mimesi platonica, applicata a tutte le attività umane, la mimesi è, secondo Aristotele, imitazione e rifiuta il mondo delle idee.

Il concetto platonico di mimesi è molto significativo per questo rispetto. Nelle due fonti che parlano di mimesi, Symposion e il decimo libro della Repubblica, Platone difende due posizioni diverse che partono entrambe dalla stessa ontologia. Come è noto, per Platone gli attributi sono reali solo per le idee e di conseguenza il mondo fenomenico è l'imitazione delle idee. "I poeti sono bugiardi" afferma Platone nel Libro $X$ della Repubblica, e non ha completamente torto, anche se dal suo punto di vista, dalla sua ontologia, intendeva vituperare quella che considerava una falsificazione della realtà (Spang, 1984, p. 153). Il filosofo ha ragione, perché tutta la creazione letteraria è in misura maggiore $\mathrm{o}$ minore un rimodellamento della realtà. Tutta la creazione letteraria implica necessariamente l'incarnazione più o meno libera di una realtà umana e materiale, che non è - salvo in poche eccezioni riproduzione servile di una realtà esistente. Il problema è se questa è una bugia o no.

Per Platone la mimesi è un'imitazione delle apparenze (di ciò che è dato sensualmente) e in questo senso l'imitazione collocata differisce dal terzo grado che la separa dalla verità. Il filosofo usa la metafora di un tavolo e di un letto per giustificare il suo punto di vista. Il vero essere è concesso solo alle loro stesse idee (e quindi a ciò che il tavolo e il letto sono nella loro essenza). In secondo luogo, possiamo parlare di tavoli e letti fatti reali, diversi e numerosi da un falegname. È solo in terzo luogo che percepiamo l'opera mimetica (la mimica "modello" è legata alla pittura, e quindi ad altri tipi di imitazione). Il pittore non fa né un tavolo né un letto, ma solo il loro aspetto. Pertanto, non 
può rappresentare la natura stessa del letto, quindi falsifica la realtà. Inoltre, inganna il destinatario stesso: gli mente (Januszkiewicz, 2002, p. 142).

Il Simposio parte dal concetto che i fenomeni sono innescati da idee e quindi hanno un valore cognitivo e possono servire come mezzi di approccio alle idee. C'è però una barriera, solo il bello è capace di mediare tra fenomeni e idee, e purtroppo per i letterati Platone non considera che il suo lavoro sia legato alla creazione del bello. Nella Repubblica Platone insiste sulla discrepanza tra fenomeni e idee, sottolineando il fatto che l'imitazione è necessariamente carente in quanto può essere eseguita solo a costo di una notevole perdita di sostanza rispetto alla pienezza delle idee. È un pensiero di Platone piuttosto che di Aristotele intendere la mimesi come una copia. In Platone la mimesi è intesa come una copia perché non c'è continuità tra il mondo ideale e il mondo sensuale: il mondo ideale è reale, mentre il mondo sensuale è una pallida somiglianza. Prendendo come modello le cose sensibili, l'arte diventa una copia di una copia (Malo, 1992, p. 316). Come dice Giangiacomo Vale (2014, p. 91):

La riflessione aristotelica sulla poesia, allo stesso modo della riflessione platonica, trova nel concetto di mimesis il suo nodo centrale. Aristotele concorda pienamente con Platone sulla essenza dell'arte come imitazione. I due filosofi si pongono tuttavia su posizioni opposte riguardo alla valutazione dello statuto epistemologico della mimesis: se per Platone essa segna la doppia distanza dell'arte dalla verità (dall'Idea), determinandone l'impotenza conoscitiva e collocandola sul piano della doxa, Aristotele, «eliminata la trascendenza dell'Idea e tolta di conseguenza ogni valenza ontologica alla nozione di imitazione», la considera invece fonte di vera conoscenza. Questo fondamentale rovesciamento è il presupposto indispensabile ed imprescindibile che porta l'allievo a sviluppare una teoria che contraddice radicalmente il maestro, pur non preoccupandosi di criticarne esplicitamente la concezione. Le due opposte posizioni poggiano evidentemente su una diversa considerazione del mondo sensibile imitato. Se per Platone questo è a sua volta un'imitazione della vera realtà, Aristotele, in un generale contesto teorico che rifiuta la metafisica platonica, considera invece la natura imitata dall'arte realtà a tutti gli effetti, per cui una sua imitazione non può che produrre "vera" conoscenza.

Infatti, come si legge in Poetica, secondo Aristotele, due ragioni stanno all'origine dell'arte poetica e ne determinano la veridicità: l'atteggiamento, l'istinto umano da seguire per acquisire conoscenza e piacere da questa azione (Aristotele, Poetica, IV 1448b, pp. 4-20): 
Due sembrano essere, in generale, le cause che hanno dato origine alla poesia; e tutte due sono proprie della natura umana. La prima causa è questa. L'imitare è un istinto di natura comune a tutti gli uomini fino dalla fanciullezza; ed è anzi uno dei caratteri onde l'uomo si differenzia dagli altri esseri viventi in quanto egli è di tutti gli esseri viventi il più inclinato alla imitazione. Anche si noti che le sue prime conoscenze l'uomo le acquista per via di imitazione; e che dei prodotti dell'imitazione si dilettano tutti. [...] E il motivo è questo, che l'apprendere non è solamente per i filosofi un piacere grandissimo, ma anche per gli altri uomini alla stesso modo; [...] il diletto che proviamo a vedere le immagini delle cose deriva appunto da ciò, che, attentamente guardando, ci interviene di scoprire e di riconoscere che cosa ogni immagine rappresenti [...]. La seconda causa è questa.

Platone osserva che l'uomo si compiace di imitare. La mimesi entra così a pieno titolo nella sfera etica: sarà valutata come benefica o dannosa in relazione al piacere che produce. Platone crede che il piacere dell'arte sia puro - indolore - e quindi superi il piacere fisico. Tuttavia, poiché l'imitazione ci distrae dal contemplare le idee, questo piacere è visto come qualcosa di negativo. La Repubblica sembra accettare solo i poeti che ci permettono di incorporare i tropos o paradigmi sociali-filosofo, guerriero e contadino nella loro perfetta purezza; quindi è preferibile che i cittadini incarnino tropos corrispondenti al loro status sociale. In definitiva, la poesia, che è un'imitazione e quindi prende le distanze dalle idee, si libera dai propri difetti quando si tratta di servire le idee.

Invece, Aristotele intende l'arte come una continuatio naturae, cioè come un'estensione della realtà. Il mondo reale è sia sensibile che comprensibile, ma l'uomo, essendo razionale, non è soddisfatto del senso naturale e vuole che la natura gli assomigli in qualche modo. Da questa insoddisfazione nascono l'arte culinaria, l'arte della casa e dell'abbigliamento, le belle arti. L'imitazione di Aristotele non è una semplice ricreazione della natura, ma la scoperta di ciò che può essere migliorato nella natura, non in un processo naturale, ma dall'azione umana; quindi l'uomo scopre possibilità trovate in natura che aspettano la sua realizzazione (Malo, 1992, p. 317).

La novità del concetto di mimesi di Aristotele potrebbe essere riassunta in una frase: "imitazione" non è riflessione, proiezione, copia. Non è difficile notare che questa comprensione del mimetismo è polemica rispetto all'approccio proposto da Platone. In primo luogo, lo Stagirita ritiene che "il compito del poeta non è quello di presentare eventi reali, ma quelli che potrebbero accadere, essendo la possibilità basata su probabilità e necessità" (Aristotele, Metafisica, 985b). La rappresentazione ("mimetizzazione”) si basa 
sulla probabilità e sulla necessità. La probabilità non è la stessa della verità. Quest'ultimo è di fatto, quindi, ciò che è oggettivamente verificabile. Il brano sopra citato è seguito dal famoso confronto tra poesia e storia. La storia ci racconta cosa è successo. In questo senso, è costretta ad adottare una prospettiva dettagliata e individuale. Altrimenti la poesia tende alla filosofia: racconta cosa può accadere. La poesia usa una generalizzazione attraverso la quale si dovrebbe comprendere questa probabilità e necessità, e il fatto che la poesia non è interessata a nessuna unità specifica (Januszkiewicz, 2002, p. 143).

In secondo luogo, il mimetismo non è sinonimo di mappatura, perché quando si tratta di un'opera, il destinatario non ha bisogno della conoscenza di un riferimento, un oggetto non artistico che servirebbe da modello per ciò che sta imitando. Se assumiamo che "l'imitazione" nell'arte non sia altro che la copia, dobbiamo convenire che la misura del valore artistico di un'opera è la sua fedele adesione (somiglianza) con la realtà imitata. In altre parole, la misura di un'opera è la stessa realtà (non artistica). Nel frattempo, Aristotele, argomentando con la convinzione sopra espressa, propone due tesi estremamente importanti:

- nel trattare un'opera d'arte, possono verificarsi situazioni in cui il destinatario non è in grado di decidere in che misura vi sia una somiglianza tra l'imitato; L'oggetto presentato nell'arte può essere completamente sconosciuto allo spettatore. Ciò non significa, tuttavia, che l'esperienza dell'arte debba essere incompleta in questo caso,

- lo stesso oggetto (evento), a seconda che lo incontriamo nella realtà $\mathrm{o}$ in un'opera d'arte, spesso evoca in noi reazioni diverse, co-crea due esperienze diverse: "Ci sono, dopotutto, cose come l'apparizione degli animali più sgradevoli o cadaveri, che guardiamo con un sentimento di dolore, ma con piacere guardiamo le loro immagini particolarmente fedeli" (Aristotele, Poetica, IV, 1448b). In altre parole: l'esperienza dell'arte è completamente autonoma, unica nel suo genere,

- terzo, il fatto che la mimesi non sia mera copia può essere visto dal modo in cui Aristotele parla dell'errore: "Si dice: il poeta ha sbagliato perché ha presentato cose impossibili. Intanto è lecito se, grazie a questo, raggiunge l'obiettivo proprio dell'arte (...) quindi se per questo l'una o l'altra parte dell'opera diventa più toccante" (ivi). Le dichiarazioni citate ci fanno immaginare la mimesi come un contrario di "copiare". 
In Poetica, Aristotele si dedica a riflessioni sulla poesia, soprattutto tragedia, e in misura minore epica. È noto, tuttavia, che il lavoro non è completo: molto probabilmente, la parte comica è andata persa. Il punto di partenza era l'assunto del filosofo che le arti mimetiche differiscono in termini di mezzi, oggetti e modo di imitazione. Il primo includeva ritmo, parola e canto; tra gli elementi ha distinto la trama, i personaggi e il modo di pensare dei personaggi. Pertanto, gli oggetti di imitazione sono le azioni umane, che sono il risultato del pensiero (dianoia) e dei caratteri (ethos) dei singoli personaggi. Il pensiero si manifesta nelle parole e i personaggi nei fatti.

$\mathrm{Va}$ notato che i personaggi possono essere rappresentati da tre tipi (a causa del soggetto della raffigurazione) di mimesi: idealizzante (quando mostriamo qualcuno migliore di quello che realmente è), caricaturale (quando presentiamo qualcuno peggio di loro) e realistico (quando mostriamo a qualcuno com'è). Aristotele sottolinea, tuttavia, che l'oggetto più importante della mimesi non sono tanto le persone in quanto tali, ma la trama stessa, cioè il sistema ordinato degli eventi. Infine, quando si tratta del modo di imitare, il filosofo ha in mente, nel caso della tragedia e della commedia - uno spettacolo, e nel caso di un'epica - epos. Questa differenziazione deriva, in generale, da diverse forme di segnalazione: drammatica (tragedia, commedia) ed epica (poema epico). A questo proposito, a sua volta, dobbiamo prestare attenzione al verificarsi di due tipi di mimesi: drammatica e diegematica.

Nella Repubblica, Platone individua gli elementi costitutivi della poesia in metro e melodia senza i quali, a suo avviso, diventa irrilevante, se non disgustosa. Al contrario, secondo Aristotele, la componente fondamentale ed essenziale della poesia non è la forma (léxis), ma il contenuto, cioè il racconto (mitos), consistente nell'imitazione (mimesi). Tuttavia, questa non è una mimesi delle persone: le persone sono certamente imitate, ma non nella misura in cui hanno un carattere o un altro (ethos) che si basa sulle qualità morali dei personaggi, sulla virtù o l'immoralità di ciò che fanno o dicono. Come dice Aristotele (Poetica, XVI, $1454^{\circ}$, pp. 15-24):

(...) la tragedia non è mimèsi di uomini, bensì di azione e di vita, che è come dire di felicità e [...] infelicità [le quali] si risolvono in un'azione, e il fine stesso [della vita, cioè la felicità] è una specie di azione, non una qualità. Ora gli uomini sono di questa o quella qualità se considerati rispetto al carattere, ma rispetto alle azioni sono felici o infelici. Non dunque i personaggi di un'azione drammatica agiscono per rappresentare determinati caratteri, ma assumono questi caratteri [...] a cagione dell'azione. D'onde segue che il complesso dei casi, ossia la favola, 
è $[\ldots]$ il fine della tragedia; [...] senza azione non ci potrebbe esser tragedia, senza caratteri si.

In Poetica, da un lato, si dovrebbe distinguere la dimensione ricettivaaffettiva della mimesi e, dall'altro, la dimensione ricettiva-pragmatica. L'aspetto affettivo della mimesi va inteso come la convinzione espressa in Poetica, del tutto evidente nella storia dell'estetica, che un'opera d'arte influenzi i sentimenti di chi la riceve. Il piacere che deriva dal comunicare con un'opera del genere è di derivare dalla natura stessa della rappresentazione imitativa nell'arte. Secondo Aristotele, gli elementi più importanti di una tragedia che adempie a questa funzione sono le componenti della trama: vicissitudini e riconoscimento. Sarebbe meglio che questi due elementi fossero in relazione tra loro, perché allora sono in grado di evocare sentimenti di pietà e paura. Allo stesso tempo: "Ci dispiace per la sfortuna di un uomo innocente, ma temiamo la sfortuna di un uomo che è come noi". La dimensione pragmatica ricettiva della mimesi trova la sua giustificazione nella convinzione di Aristotele che le imitazioni possano riguardare non solo la realtà reale o ideale, ma anche "come si dice o si pensa che sia".

La conclusione che si può trarre dalle considerazioni precedenti è che il fatto già riconosciuto dai ricercatori è giustificato: il mimetico in senso aristotelico non ha nulla a che fare con la copia o la mappatura. Piuttosto, dovrebbe essere considerato come sinonimo del concetto di finzione letteraria negli studi letterari. Il termine verosimiglianza invece non designa, come ho indicato all'inizio, un'altra forma di creazione di realtà letterarie, ma una sorta di regolatore, di misura più o meno severa a seconda del tempo in cui viene applicato, ideato originariamente per mantenere il difficile equilibrio tra realtà letteraria e straordinaria, tra imitazione e invenzione. Mimesis e finzione costituiscono due modi per creare la realtà letteraria sulla base di elementi della realtà esistente: non si escludono a vicenda, ma anzi si completano a vicenda, la loro differenza è graduale e non essenziale.

\section{La metafora come mimesi perfettiva}

La specificità della mimesi eseguita dalle arti visive è sottolineata in diversi frammenti di Poetica. In una di esse, Aristotele sottolinea che il poeta deve imitare i bravi ritrattisti che "dipingono per ricreare le peculiarità dell'originale, componendo ritratti simili, ma esaltandone la bellezza". Secondo Stagirita, il primo tipo, la somiglianza imperfetta, corrisponderebbe nel caso della pittura a una semplice riproduzione fotografica, e nel caso della poesia, a una 
narrazione fedele, cioè alla messa in scena di eventi (Aristotele, Poetica, 15, 1454b, p. 10). Il secondo tipo, invece, la somiglianza che esalta, corrisponderebbe nel caso della pittura a un ritratto che, attraverso le caratteristiche fisiche esterne del protagonista, ci permette di scoprire il suo temperamento o carattere; nella poesia questo tipo di somiglianza si può trovare nella tragedia, dove i personaggi drammatici attraverso le loro azioni, gesti e parole rappresentano archetipi o schemi ideali.

Per parlare di metafora nel contesto della mimesi, è conveniente fare riferimento all'interpretazione ricœuriana della mimesi che sorge nel contesto di una teoria della metafora, così che, come vedremo, tutta la mimesi avrà qualcosa di metafora e tutta la metafora in metafora mimetico. Recupero di una parte della mimesi aristotelica rifiuto dell'interpretazione della mimesi come semplice copia, a partire dall'identificazione di quei tratti che la separano da essa (Martínez Sánchez, 2016, p. 131).

In primo luogo, Ricoeur sottolinea le differenze tra il concetto platonico e quello aristotelico. Platone non applica solo la mimesi a tutte le arti, ma alle cose nel loro insieme, designando vari gradi di somiglianza, mentre Aristotele ne limita l'uso alle scienze poetiche, escludendo così il teorico e il pratico. Ciò che caratterizza invece la mimesi aristotelica non è il rapporto di somiglianza (come in mimesi-copia), ma un processo di costruzione, la costruzione della trama, del mito. La mimesi come processo di costruzione si oppone al carattere passivo della nozione di copia del significato dinamico della poiesis, che la colloca anche nel campo della prassi: "non c'è più mimesi di dove c'è un fare" (Ricoeur, 1980, p. 61). Come si vedrà in Tempo e racconto, i miti, come la mimesi, non sono intesi come una struttura, ma come una "operazione" (Ricoeur, 1987a, p. 83).

Imitare, in questo senso, non è duplicare la realtà, ma ricomporla, rifarla, in modo che la vicinanza alla realtà umana, che è ciò che viene imitato, si unisca alla distanza imposta dalla costruzione della trama. La dialettica vicinanza/distanza, che esprime il paradosso della mimesi, ci offre la migliore prospettiva per comprendere la proposta ricœuriana, mentre la vicinanza indica la dimensione referenziale e la distanza il momento dell'invenzione-finzione.

Infine, la seconda caratteristica che l'autore conserva nell'interesse della sua ricerca è l'idea di elevazione, che implica una riduzione della mimesi a mimesi tragica: "La seconda caratteristica che interessa la nostra ricerca è così affermata: nella tragedia, a differenza della commedia, l'imitazione delle azioni umane è un'imitazione esaltante. Questa particolarità è la chiave per comprendere la funzione della metafora" (Ricoeur, 1980, p. 63). 
La ricomposizione mimetica, quindi, ha un tratto specifico nella tragedia: è un'imitazione che esalta, esalta o eleva ciò che imita, in questo modo la struttura della mimesi coincide con quella della metafora, poiché in essa c'è anche la unione della ricomposizione con l'elevazione e del richiamo alla realtà (vicinanza) con l'invenzione (distanza). La combinazione di questi elementi e la tensione che implicano definiscono la funzione referenziale della metafora. L'elevazione che Aristotele applicava alla tragedia è intesa da Ricœur come elevazione di significato, che a sua volta è assimilata allo spostamento di significato proprio della metafora, così che la mimesi è interpretata dalla metafora e la metafora dalla mimesi. Si stabilisce così un parallelismo tra la metafora e il mito, o trama, così che lo spostamento di significato costitutivo della metafora è visto come l'elevazione di significato operata dalla mimesi attraverso la costruzione della trama. Il parallelo si estende alla "katharsis", considerata come elevazione del sentimento (Martínez Sánchez, 2016, p. 134).

Nella composizione della trama, la natura imitata è l'azione umana. Il rapporto mimetico tra arte e natura, tra il poema tragico e l'azione, non mina l'autonomia dell'arte, né la riduce a mera copia, ma anzi ne stabilisce la dimensione referenziale (la mimesi garantisce che il mito ci racconta la realtà ). Il riferimento qui va inteso nel senso della nostra appartenenza al mondo: l'essere nel mondo è l'orizzonte di ogni mimesi, soprattutto nella sua accezione dinamica e creativa. Questa mimesi rivela la capacità cognitiva dell'immaginazione e introduce una forma di verità nella poesia come potere di rivelazione ontologica, come rivelazione del reale, in modo che al vederecome dell'affermazione metaforica corrisponde un essere-come nell'ordine extralinguistico. La nozione di mimesi implica quindi una teoria della verità, che inizialmente sorge come verità metaforica e si discosta dalla concezione di adeguatezza della verità propria della nozione di rappresentazione.

Se una tecnica è sufficiente per ottenere una fedele imitazione, allora non è sufficiente ottenere una somiglianza che affini la realtà: oltre agli aspetti tecnici che si possono apprendere, è necessario scoprire le somiglianze che questa realtà ha con le altre e che allo stesso tempo ne costituiscono l'essenza individuale, e la elevano alla categoria del modello ideale. Pertanto, Aristotele afferma che la metafora non può essere appresa perché presuppone "una buona percezione di un rapporto di somiglianza" (Poetica, 22, 1458b) e non ci sono prescrizioni per ottenerla. In Poetica la metafora è vista come la migliore fonte per imitare un modello, abbellirlo o degradarlo trasponendo realtà superiori o inferiori dal punto di vista estetico. In questo modo, la perfezione artistica si ottiene attraverso la comunicazione o il trasferimento di qualità da un essere 
all'altro. Questi tipi di metafore sono di natura estatica e corrispondono all'imitazione che viene eseguita anche attraverso il ritratto.

Tuttavia, esiste un altro tipo di metafora in cui la perfezione del modello non si ottiene attraverso la comunicazione tra le realtà, ma attraverso la rivelazione contenuta in una data realtà. In Retorica, lo Stagirita si riferisce ad esse quando afferma che le metafore più malleabili sono quelle che sensibilizzano gli oggetti o li mettono davanti agli occhi perché li rappresentano in azione; ad esempio, "dire che una brava persona è un quadrato è una metafora perché entrambi sono perfetti, ma non l'azione; dire invece che ha un vigore fiorente è un'azione" (ivi). Secondo Aristotele, la metafora dinamica contiene una maggiore perfezione perché, oltre a stabilire le somiglianze di questa realtà perfetta, ci permette di visualizzare quella perfezione in sé.

Tornando a Ricoeur, lui separa la realtà del destinatario perché distingue tra due tipi di riferimento (linguaggio colloquiale e descrittivo e linguaggio poetico o letterario, che alla fine si estende all'arte nel suo insieme): la realtà ordinaria e la realtà che trasforma e cambia; in un certo senso, una realtà superficiale e una realtà profonda - una realtà di maggiore densità ontologica. Questa realtà è ciò che troviamo nella sua normale comprensione del mondo, questa realtà è riprodotta dalla finzione, dall'arte che in qualche modo producono. Questa idea di produzione, essenziale nei concetti di mimesi e regolazione, si confronta con l'ingenua o facile divisione tra realtà e finzione; tuttavia, entrambe le realtà sono combinate nel destinatario dell'opera, perché il mondo della sua azione non è ridotto a una realtà trasformata e la trasformazione influisce in ultima analisi sulla sua intera realtà.

Aristotele, sebbene attribuisca un'importante funzione alla metafora come strumento che consente l'imitazione poetica al massimo grado, non conclude che l'essenza della poetica sia la metafora. Ciò è dovuto, da un lato, all'identificazione quasi completa della poesia con la tragedia, mostrando scarso interesse per ciò che consideriamo poesia lirica; d'altra parte, presta poca attenzione alla poesia religiosa.

Se Stagirita analizzasse questi generi, scoprirà che sono collegati da un tentativo di esprimere l'ineffabile. Quindi né i gesti, né i balli, né il "linguaggio della tragedia" servono da veicolo, solo la metafora è uno strumento privilegiato per parlare di qualcosa che non possiamo concettualizzare perché parzialmente sconosciuta o a causa di essa questa realtà (divina) trascende tutti i tipi di esperienza sensoriale. In questo modo, la metafora diventa non solo una conoscenza analogica della realtà sensoriale, ma anche una comunicazione di realtà ineffabili attraverso realtà conosciute, significato più pieno perché il suo Principio di analogia lo supera in perfezione: i regni della terra 
raggiungono la perfezione nel regno celeste, la persona umana nelle persone divine.

Sebbene Platone ipostatizzi un'idea e la induca a trascendere la realtà significativa, l'analogia è tra aspetti della stessa realtà. Ecco perché Aristotele critica giustamente l'idealismo platonico, perché la trascendenza delle idee si realizza solo nella nostra intelligenza. Ebbene, se il mondo è veramente concepito come creato, l'analogia tra trascendenza reale e realtà contingente si stabilisce in un modo diverso da quello usato dall'astrazione, ma è anche una porta verso la trascendenza.

Una metafora basata su questo tipo di analogia ci permette di esplorare il mistero: le parole, pur mantenendo la loro semantica sincronica e diacronica, ampliano all'infinito il significato, diventando simboli del non detto. La mimesi, ottenuta attraverso una metafora, non deve quindi essere vista come una copia di un modello o anche come una perfezione puramente casuale aggiunta alla realtà, ma è una scoperta dell'essenza delle cose e un'espressione ineffabile.

\section{Tre tipi di mimesi - Mimesis-mythos-praxis}

Per parlare di tre tipi di mimesi, occorre fare riferimento al concetto di narrativa letteraria, in quanto è estremamente importante per una tale presentazione di mimesi. Le opinioni sullo status della narrativa letteraria, sia in Platone che in Aristotele, sono situate tra realismo e costruttivismo. Il realismo che si può trovare nelle opinioni di Platone presuppone che ci sia una realtà in sé a cui l'arte allude attraverso la sua imitazione. La parola "realtà" come sostantivo significa un oggetto a cui il soggetto si riferisce in qualche modo. Così, scrivere e parlare di "realtà" presuppone all'inizio un dualismo soggettooggetto. Platone ha sottolineato la natura secondaria della finzione artistica rispetto alla realtà che ha svalutato in modo significativo l'arte, che non solo non avvicina all'essere reale (il mondo delle idee), ma introduce anche a uno stato di sappunto e delusione (Ziółkowska-Juś, 2014, p. 190).

Il pensiero di Platone è stato portato avanti da sostenitori dell'arte realista e naturalistica, per i quali il compito più importante era quello di ottenere la massima compatibilità possibile della finzione con il mondo reale. Esempi di ipotesi realiste sono: la letteratura dell'Illuminismo, il romanzo positivista o la letteratura realista socialista del XX secolo. Il problema principale per realisti e naturalisti era la definizione della parola "realtà", che paradossalmente si è rivelata molto ambigua. 
Le opinioni dei costruttivisti, d'altra parte, si basano su un assunto completamente diverso. Il costruttivismo, già presente nelle opinioni dei sofisti, si sviluppò pienamente nel pensiero di Friedrich Nietzsche, e oggi nelle considerazioni di Richard Rorty, Stanley Fisch, Michel Foucault e altri. I costruttivisti sostengono che la nozione di "realtà" è soggettiva. Non c'è verità oggettiva in questa prospettiva, ma solo interpretazioni. In altre parole, la realtà non è qualcosa di scoperto ma qualcosa di costruito. Questa prospettiva conferisce alla letteratura e all'arte in generale un rango diverso da quello platonico. Le opinioni dei costruttivisti sono coerenti con quelle platoniche in quanto l'obiettivo di un'affermazione letteraria non è perseguire la verità, ma condurre le persone in uno stato di delusione. Tuttavia, poiché non esiste una realtà a cui l'arte possa riferirsi, il suo status subisce una trasformazione significativa. In questo approccio, l'arte non è riproduttiva. Non può più servire la verità della realtà, perché non esistono verità oggettive e realtà oggettive. L'arte produce solo l'effetto della veridicità e l'effetto della realtà, e come tale gioca un ruolo retorico, terapeutico e persuasivo.

I costruttivisti sono particolarmente vicini all'arte romantica e d'avanguardia, che enfatizza il mondo interno e soggettivo piuttosto che i suoi riferimenti esterni. Poiché tutto è un costrutto individuale o sociale, l'arte consiste principalmente nel proporre una nuova visione originale del mondo che cambierà radicalmente la sua percezione. In questo senso, l'arte dovrebbe interpretare le interpretazioni, portare all'esistenza una versione della realtà nuova, più interessante e più convincente di prima. Questa prospettiva rimuove la differenza tra uno scrittore e un lettore che legge. Chi legge non è solo un performer, interprete, ma anche un creatore nel pieno senso della parola. Secondo i costruttivisti, il significato non è una componente del testo, né fa parte dell'autore dell'opera, ma è creato appunto nell'atto della lettura, che diventa così scrittura (Ziółkowska-Juś, 2014, p. 191).

Il mondo della finzione si configura in una nuova realtà per il lettore, anche se sappiamo che la riproduzione esatta della realtà è impossibile perché "il linguaggio riflette il mondo in modo peculiare, ossimoro, come un grido silenzioso; la realtà è registrata nel verbo senza apparire in forma tridimensionale" (Gullón, 1999, p. 125). Così, osserviamo che la realtà letteraria non è una realtà isolata, ma piuttosto è incatenata a una serie di altre realtà che consentono alla storia di accadere, dando origine a un "nuovo prodotto", una volta che rivela o trasforma altre realtà. In mezzo a questa intricata ri-creazione di realtà, tra l'altro, l'opera letteraria è capace di svelare le "tombe" dell'oblio, nascoste dallo spazio o dal tempo, ma che esistono, sono esistite o esisteranno, 
modificando tutto, offrendo la possibilità di trasformazione, di rappresentazione.

Nella visione di Eco (1999, p. 11), "ogni testo è una macchina pigra che chiede al lettore di fare parte del suo lavoro". E, all'avvio di questa macchina, il lettore-collaboratore, nel suo ruolo di "operatore", ha bisogno di "controllare" la macchina, dotandosi di una conoscenza preventiva della materia in cui andrà ad interferire, senza compromettere il funzionamento della macchina e il contesto in cui è inserita. Quindi, è importante ricordare che il quadro immaginario gestisce un universo la cui logica di funzionamento manca di verità assoluta, subordinata a componenti non sempre basate su una realtà esistente. Il testo, da solo, non fornisce tutto ciò che il destinatario dovrebbe capire, che sarebbe da riassumere al minimo. Quindi, la finzione narrativa, e i suoi pilastri supportati dall'immaginazione, consentono al lettore di essere, in un certo modo, un soggetto autonomo, poiché la velocità fatale della finzione narrativa costruisce un mondo e i suoi eventi mancano di possibilità di dire tutto di lui.

Per quanto riguarda il testo narrativo, la finzione è un percorso con molte biforcazioni. Quando non conosci molto bene il percorso, non sei esente dal perderti per strada o optare per ciò che può sembrare più pratico e/o piacevole, senza esserlo. Per quanto diafano e senza molte avversità possa sembrare un percorso, per percorrere il percorso di finzione di un testo letterario è necessario essere molto attenti durante tutta la traiettoria, in modo che le decisioni prese per seguire il viaggio non presentino notevoli difficoltà. Questo porta il lettore ad essere costretto, in ogni momento, a fare scelte forti, supportate da una previa conoscenza del Mondo che servirà sicuramente da cuneo di supporto per il viaggio.

Sempre sulla base dei contributi di Eco (1999, p. 139), possiamo capire che: "nella narrativa i riferimenti esatti al mondo reale sono mescolati così strettamente che, dopo esserci abituati un po' a un romanzo, e aver confuso, com'è giusto, elementi fantastici e riferimenti alla realtà, il lettore non sa più esattamente dove si trova". Partendo dal romanticismo, la finzione mette in scena l'alterità attraverso un sé modificabile, camuffato, inscenato, dando luogo ad un sé-altro, in una dualità cosciente, frutto della complessità e del ragionamento della sua produzione, i cui antecedenti fondamentali si trovano nell'antichità, seguiti dalla trascendentalità della letteratura allegorica medievale. Il tutto supportato dai pilastri del romanzo gotico inglese del XVIII secolo che fornisce elementi più immediati della letteratura del sé-altro, che dà origine all'espressione e all'apogeo del fantastico. 
Il rapporto tra realtà e finzione è estremamente complesso. La produzione letteraria mette in scena finzione e realtà, trasformando la finzione in realtà e la realtà in finzione. Intorno a questo, Bravo (1997, p. 56) sottolinea che:

il regno della finzione esiste sempre in relazione al regno della realtà, e questo rapporto ha, in termini generali, due modi di manifestazione: nel primo, la finzione cerca di mostrarsi; cessare di esistere, in modo che il reale esista al suo posto; la finzione si identifica con un credibile che si identifica con il reale. La finzione acquisisce e così dimostra la sua ragione d'essere: esiste perché esprime il reale diventando la sua credibilità.

Nel caso della letteratura realistica, l'espressione della realtà acquista verosimiglianza. La finzione assume la condizione di reale, essendo una realtà diversa, con proprie leggi e ragioni; trasgredisce il limite che le separa, rompendosi da una zona all'altra, generando una produttività rappresentata da una delle sue principali espressioni: l'immaginazione. La creazione del nuovo mondo, diverso da quello reale, suppone una duplicazione della realtà, una creazione immaginaria, una realtà sostanziale, derivante dall'accumulo del linguaggio, da un aggettivale del mondo.

Paul Ricoeur distingue tre tipi di mimesi. Si interessa non solo della narrativa letteraria intesa isolatamente dal contesto e dall'esperienza della vita (mimesi II), ma soprattutto del mondo da cui nasce e che imita (mimesi I) e del mondo del lettore, su cui influisce in modo significativo (mimesi III). Ricoeur non vuole accontentarsi dell'analisi strutturale e semantica dell'opera, ma come ermeneuta si sforza di "ricostruire la totalità delle operazioni attraverso le quali l'opera emerge dalle profondità impenetrabili della vita, dell'azione e dell'esperienza. Dopotutto, l'opera è data dall'autore al lettore che l'accetta e cambia così la sua azione" (Ricoeur, 2008, p. 84). Distinguere tre mimesi indica che la narrativa letteraria non è una creazione autonoma, ma è subordinata all'esperienza umana e pratica. Da un lato si disconnette dal mondo della vita e, dall'altro, è radicato nella prassi quotidiana, sviluppata dall'etica e aperta al lettore, nella quale sta solo realizzando il proprio destino. L'importante è ciò che la precede (pre-comprensione), ovvero l'attività creativa dell'artista e ciò che segue (post-comprensione), ovvero l'esperienza di lettura (Melberg, 2002, p. 55).

La mimesi I riguarda la creatività poetica, le fonti della formazione poetica. È ciò che Aristotele intende per imitazione e rappresentazione. Facendo riferimento a Heidegger, Ricoeur afferma che la composizione non appare nel vuoto, ma che nasce dal radicamento umano nel mondo e da una 
pre-comprensione di natura pre-concettuale e che in realtà è una comprensione strutturata in modo diverso. Incorporando il concetto di Aristotele nel discorso ermeneutico, Ricoeur attira l'attenzione sulle radici della composizione narrativa e immaginaria nell'esperienza di vita. Vale la pena prestare attenzione alle strategie letterarie per trasformare il campo pratico in campo immaginario (Ziółkowska-Juś, 2014, p. 192).

La finzione utilizza la fenomenologia di un atto correlato a una rete di termini quali: autore, scopo, mezzi, circostanze, sostegno, ostilità, cooperazione, conflitto, successo, fallimento, ecc. (Ricoeur, 2008, p. 87). Le azioni e le esperienze con le circostanze che lo accompagnano diventano il tema della storia. La narrazione trasforma il regno della vita pratica, integrando e concretizzando la terminologia dell'azione. A causa del legame diacronico e temporale, la trama stabilisce un legame tra gli autori, l'azione, i sentimenti, le circostanze e le motivazioni dell'azione. Questa relazione nella vita pratica quotidiana a volte è difficile da afferrare.

La composizione narrativa è radicata nei significati simbolici presenti in campo pratico, il che significa che non potrà mai essere eticamente neutra. La nostra esperienza è sempre in qualche modo mediata simbolicamente prima di entrare nel regno della parola o della scrittura. La mediazione simbolica dovrebbe essere intesa come norme, valori, convenzioni, gesti, segni abbreviati presenti in una data cultura che semplificano la sequenza del ragionamento logico, così come metafore che nascondono un significato più profondo che può essere disponibile solo per la conoscenza esoterica (Ricoeur, 2002, p. 271). Questo significato simbolico primordiale consente il funzionamento, la comunicazione, la comprensione e la comunicazione. Quindi non è solo la narrazione a dare senso all'esperienza, ma piuttosto la composizione narrativa utilizza i significati simbolici presenti nell'area pratica (anche implicitamente). Il ruolo della narrativa letteraria è trasformare i significati disponibili e indicare modi di valutazione diversi, ma possibili. Le azioni umane non sono mai eticamente neutre, il che significa che la finzione di cui sono oggetto è essa stessa strettamente correlata alla valutazione. È stato già notato da Aristotele, la cui poetica si basa su alcuni presupposti etici.

Inoltre, la storia si riferisce alla strutturazione temporale della nostra esperienza pratica. Le finzioni letterarie immaginarie non rimuovono l'aporia di entrambi i tempi, ma piuttosto mostrano la loro connessione irriducibile, iscrivendo il tempo della coscienza (esperienza) nel tempo cosmico. Così, la letteratura gioca una sorta di "gioco con il tempo", rendendo quest'ultimo più docile e comprensibile per l'uomo. Si può dire che ristrutturando la nostra 
esperienza temporale, la letteratura vuole domare il tempo, la cui comprensione ci sfugge costantemente. In questo senso, la storia è favorita dalla strutturazione colloquiale del tempo, espressa in termini come: "oggi", "ieri", "domani", "dopo", "allora" ecc. La trama estrae e struttura temporaneamente ciò che è per l'esperienza umana più significativo. Raccontare il tempo (la sua struttura narrativa) lo rende più umano perché, come afferma Ricoeur (2003, p. 119), "passare attraverso la narrazione è l'innalzamento del tempo, il tempo del mondo, al tempo dell'uomo". Distinguendo la mimesi I, Ricoeur ha indicato le radici dei mondi letterari in ciò che è umano. Le storie riorganizzano e trasformano la sfera della nostra vita pratica, insieme ai suoi significati, valori e temporalità già strutturati. In altre parole, la letteratura configura e trasforma ciò che ha già una certa forma, più o meno definita, nell'attività umana.

Mimesis II è la creatività che si svolge nel campo immaginario, ovvero una rappresentazione dinamica di eventi e personaggi intrecciati in avventure. Questo tipo di distinto insieme compositivo è governato da un ordine specifico, che è il mito di Aristotele, cioè il sistema degli eventi. Ricoeur estende il modello di Aristotele di stabilire intrighi a tutte le narrazioni di fantasia. $\mathrm{Si}$ tratta di configurare un mondo immaginario sulla base dell'esperienza disponibile. Affinché la storia raccontata abbia un senso, deve essere governata da una logica specifica, deve collegare e organizzare gli eventi in un insieme razionale e coerente. Questo ordinamento fittizio - estrarre un insieme coerente dalla sequenza caotica degli eventi - è stato sottolineato anche dall'autore di Poetica. Il mito di Aristotele può essere caratterizzato dalle seguenti tre caratteristiche: completezza, integrità e grandezza appropriata. Per un "insieme immaginario" è anche importante avere un inizio, una metà e una fine, ed è un inizio e una fine narrativi, non gli eventi raccontati. Inoltre, l'azione dovrebbe essere soggetta ai requisiti di necessità e probabilità che regolano la sequenza degli eventi, eliminando il più possibile la casualità. Ciò che nella vita sembra esistere simultaneamente o sotto forma di una sequenza casuale, l'intrigo lo collega e lo organizza in un insieme sensibile. Pertanto, la storia fa emergere e mostra connessioni tra personaggi, azioni, circostanze ed eventi che sono spesso impercettibili nella vita. (Ziółkowska-Juś, 2014, p. 193).

L'estetica del romanzo, trasformando l'ordine delle considerazioni etiche, privilegia l'azione e l'azione rispetto ai personaggi (personaggi), perché - come scrive Aristotele (Poetica, 22, 1460)

Lo scopo dell'imitazione è rappresentare un'azione, non le proprietà di un personaggio. Alcune caratteristiche sono associate al loro carattere, mentre le loro azioni decidono del loro successo o sfortuna [...]. Quindi l'obiettivo 
di una tragedia è il corso degli eventi, cioè la trama, e l'obiettivo è la cosa più importante in ogni cosa. La tragedia non può esistere senza azione, ma può esistere senza personaggi.

La storia mostra i personaggi come intrappolati nel contesto della propria storia, eventi, circostanze e azioni di altre persone. Una tattica importante utilizzata nella costruzione della storia è l'universalizzazione, che eliminando la casualità episodica consente di concentrarsi su ciò che è essenziale. Questa trasposizione estetica generalizza e caratterizza azioni, personaggi ed eventi, consentendo una comprensione più profonda dell'esperienza umana. Non si tratta affatto di svalutare un'esperienza specifica e individuale, ma piuttosto di farne emergere il significato. Si può dire che Aristotele e Ricoeur invertono la mimesi di Platone. L'arte idealizza. Allo stesso tempo, però, va aggiunto che gli universali rivelati nell'arte non sono idee platoniche, poiché derivano dalla saggezza pratica legata all'etica. L'arte letteraria non solo non toglie nulla alla realtà a cui si riferisce, ma ne consente anche una più profonda comprensione. Non c'è alcun riferimento tra il mondo rappresentato nell'opera letteraria e il campo dell'esperienza pratica. La creatività si allontana dalla realtà su un livello per ritornarvi su un altro livello.

La strategia del racconto, però, non consiste nell'estrarre dalla vita un ordine indisturbato, ma nel mostrare "crepes", situazioni limite, contraddizioni e discontinuità dell'esistenza umana. Ricoeur definisce una configurazione fittizia come una "sintesi di non omogenea" o "incompatibilità compatibile", sottolineando così che l'ambientazione fittizia della trama mostra e fa emergere le contraddizioni e le aporie della nostra esperienza quotidiana. Si scopre che la logica (coerenza, completezza) della storia non rimuove le difficoltà associate alla situazione di una persona di fronte a un groviglio di eventi casuali. Ad esempio, la tragedia di cui scrive Aristotele trasforma sentimenti violenti di condanna in sentimenti di pietà e paura verso l'inevitabilità delle azioni dell'eroe tragico, presentate nella narrativa letteraria, in circostanze al di fuori del suo controllo. Il disaccordo concordante (o accordo contraddittorio) si manifesta nella tragedia nel cambiamento inaspettato del destino (felicità in sfortuna o viceversa), in eventi che sono terrificanti e suscitano pietà e paura, a causa della sorpresa, in affetti violenti o in episodi occasionali. La letteratura, sebbene raccolga le contraddizioni e i dilemmi presenti nella vita, non pretende di eliminarli. Al contrario: vuole universalizzare le contraddizioni dell'esistenza, mostrarle come inseparabili dalla condizione umana.

Mimesis III si svolge all'incrocio tra il mondo della narrativa e il mondo del lettore. È anche un "luogo" dove avviene la verità della finzione, 
riconoscibile e compresa a parole. Leggere è creare e scoprire. Lo stesso Ricoeur descrive questa unità di creazione e scoperta come un concetto prospettico di verità, sottolineandone così il carattere dinamico e creativo. Più di quanto già noto viene riconosciuto e compreso. La verità dell'arte non è contenuta nell'opera finita (creazione), né nella sua pronta interpretazione, perché non può essere chiusa in un concetto, né può essere reificata in alcun modo. Se la verità dell'arte potesse essere sostituita da un concetto, l'arte ne sarebbe solo una rappresentazione esemplare. Un'idea simile è espressa in Theodor W. Adorno (1994, p.123) come segue:

Il gioco non è determinato dall'entità partecipante; ha il suo modo di essere. Il gioco è un'esperienza che trasforma i suoi partecipanti. Sembra che il soggetto dell'esperienza estetica non sia colui che suona. Ma cosa "succede" durante il gioco. In un senso simile, parliamo di un gioco di onde, luce, parti di macchine e persino parole.

In questo "gioco", il testo e il lettore diventano partner a pieno titolo. Sia il testo incontra il lettore sia il lettore va al testo. Nel processo di lettura è impossibile tracciare una linea netta tra il testo e il lettore, perché la lettura è un'esperienza di alterità che il lettore scopre dentro di sé. Si tratta qui di andare oltre il binario, come: soggetto - oggetto, soggettivismo - oggettività, interno - esterno, ecc. Il testo si apre al lettore e il lettore al testo, quindi entrambe le parti vanno oltre se stesse, trascendendo la loro vicinanza. Grazie al lettore, il testo entra nel mondo reale e il lettore, grazie alla sua lettura, entra nel mondo immaginario. L'esperienza di lettura si svolge nello spazio tra il testo e il lettore.

Leggendo, il lettore allarga i propri orizzonti. Lottando con il testo, alla fine lotta con se stesso. Si può concludere che non solo l'opera imita la sfera della vita pratica (mimesi I), ma anche il lettore imita il mondo immaginario (mimesi III), il che significa che la lettura non può essere innocente e assiologicamente neutra. L'atto della lettura, legato alla costruzione della propria identità e autocomprensione, appare quindi anche come compito etico. Ricoeur scrive dell'identità ipse, che è dinamica, processuale, narrativa e costruita in contrapposizione alla cosiddetta identità forte intesa sostanzialmente. La letteratura presenta al lettore vari modi possibili di vivere il mondo e valutarlo, fonde ciò che è sparso nella vita di tutti i giorni, ti permette di vedere e capire il mondo e te stesso in modo diverso, familiarizza il lettore con situazioni limite che possono suscitare paura, disperazione e paura 
nella vita reale, apre all'alterità in se stessi e negli altri, e infine aiuta a imparare a morire (Łebkowska, 2001, p. 138).

Pertanto, la comprensione che scaturisce dalla storia non si riferisce solo agli eventi passati e alla possibilità di cogliere il significato della propria vita passata, ma ha anche un senso prospettico, è associata alla cura, volgendo il lettore verso il futuro proiettato. Poiché le storie fanno uso della cosiddetta saggezza pratica, in esse sono presenti valutazioni sia estetiche che morali. In un certo senso, la letteratura si colloca tra il linguaggio dei fatti e il linguaggio del dovere. Sebbene non imponga al lettore regole e valori specifici, gli consente di scoprire nuovi modi di valutare. Mimesis III è un luogo importante, che completa in modo significativo il lavoro letterario, che non è affatto chiuso in sé, ma è aperto al lettore.

La letteratura, prendendo le distanze da ciò che è reale, necessario e fattuale, scopre il mondo nella sua opacità, aprendo l'accesso a ciò che è ambiguo, indefinito e misterioso. Il campo di riferimento dei mondi letterari non è solo l'immaginazione dell'autore, i testi stessi o altri testi, ma il mondo al suo livello nascosto al di là dei significati comuni e usati. La narrativa letteraria nasce dalla vita (mimesi I), la sperimenta nel laboratorio dell'immaginazione dell'artista (mimesi II), e poi, grazie al lettore, rinasce, trasformandola in modo significativo (mimesi III). Grazie alla distanza che esiste tra la narrativa letteraria e il mondo della vita, il lettore può entrare in un mondo potenziale e irreale, provare vari modi possibili di essere e infine prendere le distanze dal suo ego. Tutto ciò fa sì che l'atto della lettura trasformi il lettore, permettendogli di capire che è anche autocomprensione.

\section{BIBLIOGRAFIA}

Adorno, T. W. (1994). Notas de literatura I (trad. Manuel Sacristán). Barcelona: Ariel.

Aristotele (1964). Poetica. (trad. it. di M. Valgimigli). Bari: Laterza.

Arystoteles (1983). Poetyka. Wrocław: Ossolineum.

Eco, U. (1999). Cinque scritti morali. Milano: Bompiani.

Januszkiewicz, M. (2002). O pojęciu mimesis w poetyce Arystotelesa. Sztuka i Filozofia, 21, 137-152.

Krokiewicz, A. (2000). Zarys filozofii greckiej. Warszawa: Aletheia.

Kudra, A. (2007). (Nie)moja mimesis. Acta Universitatis Lodziensis. Folia litteraria polonica, 9 , 293-300,

Łebkowska, A. (2001). Między teoriami a fikcją literacką. Kraków: Universitas.

Malo, A. (1992). La mimesi e la metafora nella poetica di Aristotele. Acta Philosophica, 1, 316-324.

Martínez Sánchez, A. (2016). Invención y realidad. La noción de mímesis como imitación creadora en Paul Ricoeur. Diánoia, vol. 51, no. 57, 131-166. 
Melberg, A. (). Mimesis Platona, „Pamiętnik Literacki” XCII, z. 2, 2002.

Mitosek (). Mimesis. Zjawisko i problem, Warszawa 1997,

Reale, G. (1975). Platone e Aristotele, in ID., Storia della filosofia antica, Milano, „Vita e Pensiero", 1975, vol. II.

Reale, G. (1996). Historia filozofii starożytnej. Platon i Arystoteles (t. II, tłum. E. I. Zieliński). Lublin: KUL.

Ricoeur, P. (1980). La metáfora viva. Madrid: Cristiandad.

Ricoeur, P. (1985). Egzystencja i hermeneutyka. Rozprawy o metodzie. Warszawa: PAX.

Ricoeur, P. (1987). Tiempo y narración I. Madrid: Cristiandad.

Ricoeur, P. (2003). Narracja, tożsamość i czas. Kraków: Universitas.

Ricoeur, P. (2008). Czas i opowieść, t. 1. Kraków: Wydawnictwo UJ.

Spang, K. (1984). Mimesis, ficción y verosimilitud en la creación literaría. Anuario Filosófico, 17, 153-159.

Tatarkiewicz, W. (1979). Storia dell'estetica, I: L'Estetica antica. Torino: Piccola Biblioteca Einaudi.

Tatarkiewicz, W. (1960). Historia estetyki, 1.1, Estetyka starożytna. Wrocław-Kraków: PWN

Vale, G. (2014). La rappresentazione oltre la realtà. Mimesis e conoscenza teoretica nella teoria poetica aristotelica. Rivista internazionale di filosofia online, 17, 89-122.

Ziółkowska-Juś, A. (2014). Prawda fikcji literackiej w świetle hermeneutyki Paula Ricoeura. Diametros, 42, 290-313. 